\title{
Elasticity of cross-linked semiflexible biopolymers under tension
}

\author{
Alice von der Heydt, ${ }^{1, *}$ Daniel Wilkin, ${ }^{1}$ Panayotis Benetatos, ${ }^{2}$ and Annette Zippelius ${ }^{1,3}$ \\ ${ }^{1}$ Institute for Theoretical Physics, Georg-August University of Göttingen, Friedrich-Hund-Platz 1, 37077 Göttingen, Germany \\ ${ }^{2}$ Department of Physics, Kyungpook National University, 80 Daehak-ro, Buk-gu, Daegu, 702-701, Korea \\ ${ }^{3}$ Max Planck Institute for Dynamics and Self-Organization, Am Fassberg 17, 37077 Göttingen, Germany
}

(Received 5 May 2013; published 3 September 2013)

\begin{abstract}
Aiming at the mechanical properties of cross-linked biopolymers, we set up and analyze a model of two weakly bending wormlike chains subjected to a tensile force, with regularly spaced inter-chain bonds (cross-links) represented by harmonic springs. Within this model, we compute the force-extension curve and the differential stiffness exactly and discuss several limiting cases. Cross-links effectively stiffen the chain pair by reducing thermal fluctuations transverse to the force and alignment direction. The extra alignment due to cross-links increases both with growing number and with growing strength of the cross-links, and is most prominent for small force $f$. For large $f$, the additional, cross-link-induced extension is subdominant except for the case of linking the chains rigidly and continuously along their contour. In this combined limit, we recover asymptotically the elasticity of a weakly bending wormlike chain without constraints, stiffened by a factor of 4 . The increase in differential stiffness can be as large as $100 \%$ for small $f$ or large numbers of cross-links.
\end{abstract}

DOI: 10.1103/PhysRevE.88.032701

PACS number(s): 87.10.Pq, 87.15.La, 82.37.Rs, 36.20.Ey

\section{INTRODUCTION}

Many important biopolymers, such as DNA, the cytoskeletal filaments [filamentous (F-)actin, microtubules, intermediate filaments], as well as collagen in the extracellular matrix are fluctuating macromolecules with a bending stiffness intermediate between that of a random coil (Gaussian chain) and a rigid rod. Polymers whose elastic behavior is dominated by their bending rigidity are known as semiflexible. Numerous experiments probing their elasticity have become available [1,2] with the advances in single-molecule manipulation, particularly for DNA. Intriguing and qualitatively novel mechanical behavior arises if semiflexible polymers are pairwise permanently crosslinked. The elasticity of cross-linked biopolymers is widely studied experimentally via force-extension measurements. In this article, we study analytically the force-extension relation of an irreversibly cross-linked pair of semiflexible polymers within a mesoscopic theoretical model.

Ubiquitous as extracellular mechanical support is the connective-tissue protein collagen, whose fibrils achieve their strength via covalent intermolecular cross-links between triple-helical molecules [3,4]. Atomic force microscopy [5-7] has been used to analyze single collagen fibrils, which themselves consist of many microfibrils and hence can be modeled as anisotropic networks of irreversibly cross-linked semiflexible polymers [8]. Cell shape and stability is provided by the actin cytoskeleton, a network of cross-linked F-actin ranging in morphology from a dilute mesh to bundles of parallel filaments [9]. The special elastic properties due to cross-linking, closely related to biological function, thus have become a subject of increasing interest and vigorous research activity [10]. Yet the theoretical understanding is incomplete and explanations based on semimicroscopic descriptions are rare.

Crucial experimental results such as the strong stretching of double-stranded DNA [11] have been successfully explained

*heydt@ theorie.physik.uni-goettingen.de by the theoretical force-extension relation for a weakly bending wormlike chain [12]. The wormlike chain (WLC) [13-15] maps the conformations of an inextensible semiflexible polymer to one-dimensional paths whose statistical weight penalizes curvature and is determined by two length scales only: the total contour length $L$ and the directional correlation or persistence length $L_{p}$, proportional to the bending rigidity $\kappa$. The weakly bending approximation of a WLC [12] simplifies the analytical treatment by assuming that the tangent vector at any arc-length position and the end-to-end vector make a small angle. This approximation applies to polymers with a large persistence length $L_{p}$ (compared to $L$ ) or subjected to strong stretching. Inhomogeneities or intermolecular interactions have been the subject of several modifications and extensions of the weakly bending WLC: In [16], the force-extension relation of two parallel aligned, weakly bending WLCs with a single irreversible cross-link and the elasticity of an anisotropic network of aligned chains have been analyzed. Double-stranded biopolymers have been studied previously employing the railway-track model [17] or variants thereof [18]. In its simplest version, the railway-track model reveals a scale-dependent stiffness, which is renormalized on large length scales as compared to the local (bare) value [17]. In [18], a semiflexible ribbon was shown to exhibit twisted structures. In the wormlike bundle model, an arbitrary number of regularly arranged parallel filaments is effectively cross-linked by a coarse-grained, continuous interaction [19]. The effect of spontaneous polymer curvature has been studied by the authors of $[20,21]$. Weak extensibility of semiflexible polymers at strong stretching has been addressed with a combination of a WLC and a Gaussian chain, the semiflexible harmonic chain (SHC), in [22].

In this work, we consider the elasticity of two identical weakly bending WLCs connected by an arbitrary number of cross-links regularly spaced along the polymer contour. The cross-links are represented by entropic harmonic springs, which allow for a finite extent of the interpolymer distance at the cross-link sites. In the case of infinite spring strength, we obtain the limit of hard cross-links (strong topological 
constraints). By introducing infinitely many cross-links at fixed contour length, we can also model a continuous crosslinking or an attractive intermolecular interaction. The ladder structure of our system is reminiscent of the base-pair sequence of double-stranded DNA, but for the reversible hydrogen bonding. This obvious modification of our model to reversible and/or sectional cross-linking may prove versatile for future studies of, e.g., the denaturation of DNA.

The paper is organized as follows. In Sec. II, we introduce the model and the observable. In Sec. III, we present the main steps in calculating the canonical partition function, from which all equilibrium quantities can be derived. Details of this calculation are given in Appendixes A and B. In Sec. IV, we present the central result which is the force-extension relation. After presenting the general result, we particularly focus on the limit of hard cross-links, of continuous cross-linking, the linear elasticity for small forces, and the strong stretching limit. For the limit of continuous cross-linking, the general result and a short comparative discussion are given in Appendix C. We conclude and discuss further extensions of this work in Sec. V.

\section{MODEL}

We consider two weakly bending, semiflexible chains of equal bending rigidity and contour length, aligned parallel along a preferential direction $x$ and cross-linked at equidistant arc-length positions specified below. The chain configurations are described by paths $\boldsymbol{r}_{1}(s), \boldsymbol{r}_{2}(s)$, with $s \in[0, L]$ the arc-length parameter, and tangential vectors $\boldsymbol{t}_{j}(s)=\partial_{s} \boldsymbol{r}_{j}:=$ $\partial \boldsymbol{r}_{j} / \partial s$.

Our setup, first shown for space dimension $d=2$, is sketched in Fig. 1 for three cross-links. The detailed description of our approach will focus on the case $d=2$ for reasons of transparency, given that the generalization to $d=3$ is straightforward within the weakly bending approximation (without taking into account torsions). As indicated, we assume hinged-hinged boundary conditions, implying confinement of the vertical positions (here, to $y=0$ ) and vanishing curvature at the ends. These boundary conditions are motivated by the following situation: Experimentally, a tensile force can be applied via optical or magnetic tweezers that control the position of beads attached to the polymers' ends, cf., e.g., [23]. Optical tweezers usually restrict the bead's transverse motion, but not the rotation, so that no moments are exerted at the ends. Additionally, we assume $x_{1}(0)=x_{2}(0)$ to exclude an overall $x$ shift between the chains.

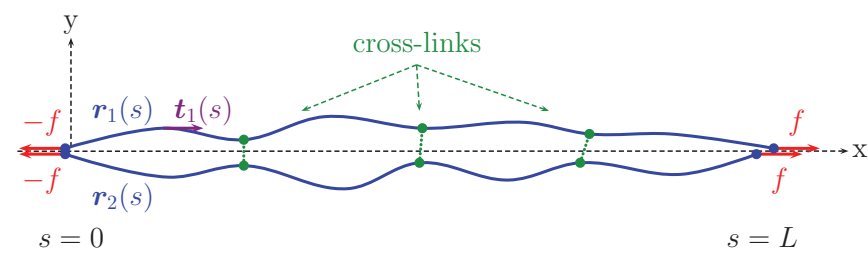

FIG. 1. (Color online) Stretched, weakly bending (see main text for explanation) chain pair connected by three cross-links.
The effective bending potential of semiflexible chains is given by

$$
\mathcal{H}_{\text {bend }}=\frac{\kappa}{2} \int_{0}^{L} d s \sum_{j=1}^{2}\left|\partial_{s} \boldsymbol{t}_{j}(s)\right|^{2},
$$

with the bending rigidity $\kappa$, related to the persistence length $L_{p}$ via

$$
\kappa=\frac{d-1}{2} k_{\mathrm{B}} T L_{p},
$$

and with the local inextensibility constraint

$$
\left|\boldsymbol{t}_{j}(s)\right| \equiv 1, \quad s \in[0, L], \quad j=1,2 .
$$

To account for inextensibility in a mathematically tractable way, we consider the weakly bending approximation: The chains' tangents preferentially align with a given direction, here $x$. A stretching force of strength $f$, acting on both ends of the chains (cf. Fig. 1), is described by the potential

$$
\begin{aligned}
\mathcal{H}_{\text {stretch }} & =-f \boldsymbol{e}_{x} \cdot \sum_{j=1}^{2}\left[\boldsymbol{r}_{j}(L)-\boldsymbol{r}_{j}(0)\right] \\
& =-f \sum_{j=1}^{2} \int_{0}^{L} d s \partial_{s} x_{j}(s)
\end{aligned}
$$

For sufficiently large stretching forces or bending rigidities, the tilt of the tangent vector away from the $x$ axis is small, so that the condition Eq. (3) reads approximately

$$
\partial_{s} x_{j}(s)=1-\frac{1}{2}\left[\partial_{s} y_{j}(s)\right]^{2}+\mathcal{O}\left(\left[\partial_{s} y_{j}(s)\right]^{4}\right) .
$$

Inserting this expansion into Eqs. (1) and (4) and discarding all but quadratic terms in derivatives of $y$, we arrive at the weakly bending approximations of the bending and stretching potentials.

Cross-links between the two chains are introduced at $N-1$ sites regularly spaced along the contours

$$
s_{b}=\frac{b L}{N}, \quad b=1,2, \ldots, N-1,
$$

dividing the contour length $L$ into $N$ sections, cf. Fig. 1 . Explicitly, we model cross-links as entropic, harmonic springs of strength $g=2 k_{\mathrm{B}} T / a_{c}^{2}$, where $a_{c}^{2}$ is the temperatureindependent squared equilibrium length of one cross-link.

Finally, the total effective Hamiltonian is

$$
\begin{aligned}
\mathcal{H}= & \underbrace{\sum_{j=1}^{2} \int_{0}^{L} d s\left(\frac{\kappa}{2}\left(\partial_{s}^{2} y_{j}\right)^{2}+\frac{f}{2}\left(\partial_{s} y_{j}\right)^{2}\right)-2 f L}_{\mathcal{H}_{0}} \\
& +\frac{g}{2} \sum_{b=1}^{N-1}\left[y_{1}\left(s_{b}\right)-y_{2}\left(s_{b}\right)\right]^{2}
\end{aligned}
$$

where $\mathcal{H}_{0}$ is the Hamiltonian of the system without crosslinking, and the last term $\propto k_{\mathrm{B}} T$ describes the entropic crosslinks in the weakly bending approximation.

Starting from the concept of harmonic cross-links at discrete sites $s_{b}=b L / N$, we will also consider the limit of continuous cross-linking, achieved by taking $N \rightarrow \infty$ and $\Delta s:=L / N \rightarrow 0$. In this case, the strength $g$ of a single 
cross-link has to go to zero, such that the total strength $\tilde{g}:=N g$ remains finite. Replacing $\sum_{b=1}^{N-1} \rightarrow \frac{N}{L} \int_{0}^{L} d s$ in the cross-link part of Eq. (7) gives a continuous, harmonic interchain attraction of strength $\tilde{g} / L$,

$$
\mathcal{H}^{(\mathrm{c})}=\frac{\tilde{g}}{2 L} \int_{0}^{L} d s\left[y_{1}(s)-y_{2}(s)\right]^{2} .
$$

It is our aim to study the effect of cross-links on the chain elasticity and hence to compute the force-extension relation exactly for an arbitrary number of irreversible cross-links. Thus, the relevant quantity is the average end-to-end extension of one chain in force direction $x$,

$$
\langle x\rangle:=\langle x(L)-x(0)\rangle_{\mathcal{H}}=L-\frac{1}{2}\left\langle\int_{0}^{L} d s\left(\partial_{s} y\right)^{2}\right\rangle_{\mathcal{H}},
$$

where $\langle\cdot\rangle_{\mathcal{H}}$ denotes the canonical average with the Hamiltonian of Eq. (7).

The force-extension relation of one weakly bending WLC without cross-links (Hamiltonian $\mathcal{H}_{0}$ ), first addressed by Marko and Siggia [12], is

$$
\frac{\langle x\rangle_{\mathcal{H}_{0}}}{L}=1-\frac{L}{2 L_{p}}\left\{\frac{\operatorname{coth} \sqrt{f_{r}}}{\sqrt{f_{r}}}-\frac{1}{f_{r}}\right\},
$$

in terms of the dimensionless variable

$$
f_{r}:=f L^{2} / \kappa,
$$

which is the ratio of stretching energy $f L$ and bending energy $\kappa / L$. The force-extension curve displays a linear regime for small forces $f$ and in the limit of strong stretching approaches the maximal end-to-end extension $L$ with a characteristic saturation $\propto f^{-1 / 2}$.

\section{PARTITION FUNCTION}

In this section, we detail the calculation of the canonical partition function, $\mathcal{Z}=\int \mathcal{D}[y(s)] e^{-\beta \mathcal{H}[y(s)]}$ (the configurational integral for both chains denoted by $\mathcal{D}[y(s)]$ ), which provides access to all equilibrium observables. For the purpose of this work, the end-to-end extension defined in Eq. (9) is obtained from $\ln \mathcal{Z}$ or the free energy [24] by differentiation with respect to the force $f$ :

$$
\langle x\rangle=\frac{k_{\mathrm{B}} T}{2} \frac{\partial \ln \mathcal{Z}}{\partial f} .
$$

The first step is to expand the chain configurations $y_{j}(s)$ in appropriate eigenfunctions. As mentioned above, we impose hinged-hinged boundary conditions, which for our system translate into $\left[y_{j}^{\prime \prime}(s):=\partial_{s}^{2} y(s)\right]$

$$
y_{j}(0)=y_{j}(L)=0, \quad y_{j}^{\prime \prime}(0)=y_{j}^{\prime \prime}(L)=0, \quad j=1,2 .
$$

According to these boundary conditions, our Fourier-series ansatz is

$$
\begin{aligned}
& y_{1}(s)=\sum_{m=1}^{M} A_{m} \sin \left(q_{m} s\right), \\
& y_{2}(s)=\sum_{m=1}^{M} B_{m} \sin \left(q_{m} s\right),
\end{aligned}
$$

with wave numbers

$$
q_{m}:=\frac{m \pi}{L}, \quad m \text { the mode number, }
$$

and $M$ the largest undulation mode considered within our continuum model (roughly, the wave-length resolution is bounded by molecular distances). With this ansatz, the Hamiltonian $\mathcal{H}$ can be written as a quadratic form in the coefficient vector

$$
\Gamma:=\left(A_{1}, B_{1}, A_{2}, B_{2}, \ldots\right)^{T} .
$$

Omitting the constant $-2 f L$,

$$
\mathcal{H}[\boldsymbol{\Gamma}]=\sum_{\ell, \ell^{\prime}=1}^{M} \Gamma_{\ell}\left[C_{\ell \ell^{\prime}}+\left(U U^{T}\right)_{\ell \ell^{\prime}}\right] \Gamma_{\ell^{\prime}},
$$

where $C$, due to $\mathcal{H}_{0}$ of the uncross-linked system, is a diagonal matrix $(\otimes$ denotes the Kronecker product),

$$
\begin{aligned}
C & =\operatorname{diag}\left(c_{1}, c_{2}, \ldots\right) \otimes\left(\begin{array}{ll}
1 & 0 \\
0 & 1
\end{array}\right), \\
c_{m} & :=\frac{L}{4}\left(f+\kappa q_{m}^{2}\right) q_{m}^{2},
\end{aligned}
$$

and $U U^{T}$ the matrix due to the cross-link Hamiltonian, cf. the second line of Eq. (7) or Eq. (8).

The partition function follows as a generalized Gaussian integral over the mode coefficients normalized by $L$,

$$
\begin{aligned}
\mathcal{Z} & =e^{2 \beta f L} \int \mathcal{D}[\boldsymbol{\Gamma}] e^{-\beta \mathcal{H}[\boldsymbol{\Gamma}]}, \\
\mathcal{D}[\boldsymbol{\Gamma}] & :=\prod_{m=1}^{M} d\left(\frac{A_{m}}{L}\right) d\left(\frac{B_{m}}{L}\right) .
\end{aligned}
$$

Returning to the end-to-end $x$ extension introduced in Eqs. (9) and (12), we wish to focus primarily on the cross-link contribution, i.e.,

$$
\langle\Delta x\rangle:=\langle x(L)-x(0)\rangle_{\mathcal{H}}-\langle x(L)-x(0)\rangle_{\mathcal{H}_{0}},
$$

since the extension $\langle x(L)-x(0)\rangle_{\mathcal{H}_{0}}$ due to thermal fluctuations of uncross-linked weakly bending WLCs only is known [12]. To that end, we write the partition function as $\mathcal{Z}=$ $\mathcal{Z}_{\text {rel }} \mathcal{Z}_{0}$, where $\mathcal{Z}_{0}$ is the partition function of the uncross-linked system and address the relative partition function [cf. Eq. (17)],

$$
\begin{aligned}
\mathcal{Z}_{\text {rel }} & :=\frac{\int \mathcal{D}[\boldsymbol{\Gamma}] e^{-\beta \mathcal{H}[\boldsymbol{\Gamma}]}}{\int \mathcal{D}[\boldsymbol{\Gamma}] e^{-\beta \mathcal{H}_{0}[\boldsymbol{\Gamma}]}} \\
& =\left[\operatorname{det}\left(\mathbb{1}+C^{-1} U U^{T}\right)\right]^{-1 / 2} .
\end{aligned}
$$

This yields the cross-link-induced extra displacement

$$
\langle\Delta x\rangle=\frac{k_{\mathrm{B}} T}{2} \frac{\partial \ln \mathcal{Z}_{\mathrm{rel}}}{\partial f} .
$$

\section{A. Finite number of cross-links}

First, we address a finite number $N-1$ of equidistant harmonic cross-links, for which the cross-link Hamiltonian is quadratic, but not diagonal in the modes. The matrix $U U^{T}$ 
is a sum of $N-1$ projectors,

$$
\begin{aligned}
U U^{T} & =\frac{g}{2} \sum_{b=1}^{N-1}\left(\boldsymbol{u}_{b} \otimes \boldsymbol{u}_{b}^{T}\right) \otimes\left(\begin{array}{rr}
1 & -1 \\
-1 & 1
\end{array}\right), \\
\boldsymbol{u}_{b}^{T} & :=\left[\sin \left(q_{1} s_{b}\right), \sin \left(q_{2} s_{b}\right), \ldots\right] .
\end{aligned}
$$

Using the identity $\operatorname{det} \exp A=\exp \operatorname{tr} A$ to expand the determinant in Eq. (21), $\mathcal{Z}_{\text {rel }}$ is accessible via traces of powers of the matrix $C^{-1} U U^{T}$,

$$
\mathcal{Z}_{\text {rel }}=\exp \left\{-\frac{1}{2} \operatorname{tr} \sum_{k=1}^{\infty} \frac{(-1)^{k+1}}{k}\left(C^{-1} U U^{T}\right)^{k}\right\} .
$$

In the trace of a power $k$ of $C^{-1} U U^{T}$, the $2 \times 2$ matrices in the Kronecker products, Eqs. (18) and (23), merely produce a factor $2^{k}$, which can be computed separately before performing the trace operation over the mode indices $m_{j}$. Hence, we are left with handling the nondiagonal mode-index structure of the projector sum Eq. (23). The corresponding matrix of rank $(N-1)$,

$$
P:=\frac{2}{N} \sum_{b=1}^{N-1} \boldsymbol{u}_{b} \otimes \boldsymbol{u}_{b}^{T}
$$

with mode indices $m_{1}, m_{2}$ has entries

$$
\begin{aligned}
P_{m_{1} m_{2}} & =\frac{2}{N} \sum_{b=1}^{N-1} \sin \left(\frac{b m_{1} \pi}{N}\right) \sin \left(\frac{b m_{2} \pi}{N}\right) \\
& =\delta_{m_{1}-m_{2}, 2 \mathbb{Z} N}-\delta_{m_{1}+m_{2}, 2 \mathbb{Z} N},
\end{aligned}
$$

where $\mathbb{Z}$ denotes the set of integers, such that $P$ is a sparse matrix of block form: In each quadratic block of dimension $2 N$, nonzero entries appear on the diagonal $(+1)$ and on one antidiagonal $(-1)$ only. For the explicit form of $P$, see Appendix A. The rows and columns display the occupation structure of the $N-1$ eigenvectors (labeled by $l$ in the following) in the Fourier basis, each with nonzero amplitudes only for a subset of modes, which at all cross-link sites are pairwise in-phase or phase-shifted by $\pi$. Modes indexed by multiples of $N$ have nodes at all cross-link sites, thus do not contribute to the cross-link energy, and constitute the kernel of $P$.

Due to this special form of the matrix $C^{-1} U U^{T}$, we are able to derive a closed expression for the trace of any power of $C^{-1} U U^{T}$ (cf. Appendix B), viz.

$$
\begin{aligned}
& \left(\frac{g N}{2}\right)^{-k} \operatorname{tr}\left(C^{-1} U U^{T}\right)^{k} \\
& =\sum_{l=1}^{N-1}\left[\sum_{\mu=1}^{\infty}\left(c_{2(\mu-1) N+l}^{-1}+c_{2 \mu N-l}^{-1}\right)\right]^{k} .
\end{aligned}
$$

Here, due to the fast decay with mode number of the inverse coefficients $c_{m}^{-1}$ from Eq. (18) (basically inverse elastic constants for the undulation modes) we have extended the summation over modes to a series. Combining Eqs. (24) and (26), we find that the relative partition function $\mathcal{Z}_{\text {rel }}$ factorizes into $N-1$ different "eigenvector" factors, or equivalently,

$$
\begin{aligned}
\ln \mathcal{Z}_{\mathrm{rel}} & =-\frac{1}{2} \sum_{l=1}^{N-1} \ln \left\{1+\frac{g N}{2} \sum_{\mu=1}^{\infty}\left(c_{2(\mu-1) N+l}^{-1}+c_{2 \mu N-l}^{-1}\right)\right\} \\
& =: \sum_{l=1}^{N-1} \ln Z_{l} .
\end{aligned}
$$

By inserting the $c_{m}^{-1}$ into the series, we obtain for the factors of the partition function, Eq. (27),

$$
\begin{aligned}
Z_{l} & =\left\{1+\frac{g L}{N f}\left[\psi_{l}(0)-\psi_{l}\left(\delta_{f}\right)\right]\right\}^{-1 / 2}, \\
\psi_{l}\left(\delta_{f}\right) & =\frac{\sinh \delta_{f}}{\delta_{f}\left(\cosh \delta_{f}-\cos \phi_{l}\right)} .
\end{aligned}
$$

Herein, we employ the dimensionless variable

$$
\delta_{f}:=\frac{L \sqrt{f}}{N \sqrt{\kappa}},
$$

which is the ratio of two lengths: The arc-length spacing $L / N$ between cross-links and the directional "memory" length $\sqrt{\kappa / f}$ of the stretched WLC, or the penetration depth of boundary conditions [20]. The dependence of the partition function on the bending rigidity $\kappa$ is via this ratio only. Additionally, we define the phases specific to the $N-1$ eigenvectors,

$$
\phi_{l}:=\frac{\pi l}{N}
$$

We note that the Gaussian statistical weights and the regular cross-link spacing simplify enormously, if not enable at all, analytical calculations.

In fact, Eqs. (27) and (28) are a central result of our paper, yielding the exact free energy of two cross-linked, weakly bending WLCs as $F=F_{0}+\Delta F$, where $F_{0}$ is the free energy of the chain pair without cross-links,

$$
F_{0}=-k_{\mathrm{B}} T \ln Z_{0}=k_{\mathrm{B}} T \sum_{m=1}^{M} \ln \frac{L^{2} c_{m}}{\pi k_{\mathrm{B}} T}-2 f L
$$

[leading to the force-extension relation Eq. (10)], and the free energy increment $\Delta F$ due to cross-links is the sum of the $F_{l}=$ $-k_{\mathrm{B}} T \ln Z_{l}$ from Eq. (28). Via $\delta_{f}$, this free energy depends on the dimensionless energy ratio $f_{r}=N^{2} \delta_{f}^{2}$ introduced in Eq. (11). As already mentioned, the use of the weakly bending approximation requires the transverse fluctuations to be small and hence that either the work done by the external force $f$ or the bending energy is large compared to the thermal energy. The dependence on the free parameter $f_{r}$ will be further discussed for the cross-link contribution to the force-extension relation. In addition, the free energy depends on the ratio of cross-link energy to stretching energy

$$
\epsilon_{g}:=\frac{g L}{N f}
$$

and, of course, on the number of cross-links, $N-1$. 


\section{B. Continuous cross-linking via harmonic interchain attraction}

Here, we sketch the derivation for an infinite number of regularly spaced cross-links $N \rightarrow \infty$ at finite total strength $\tilde{g}:=N g$, and for finite contour length $L$. With the continuous cross-link Hamiltonian Eq. (8) in normal-mode representation

$$
\begin{aligned}
\mathcal{H}^{(\mathrm{c})}= & \frac{\tilde{g}}{2 L} \sum_{m_{1}, m_{2}=1}^{M}\left(A_{m_{1}}-B_{m_{1}}\right)\left(A_{m_{2}}-B_{m_{2}}\right) \\
& \times \int_{0}^{L} d s \sin \left(q_{m_{1}} s\right) \sin \left(q_{m_{2}} s\right) \\
= & \frac{\tilde{g}}{4} \sum_{m=1}^{M}\left(A_{m}-B_{m}\right)^{2},
\end{aligned}
$$

the total Hamiltonian is diagonal with respect to the mode indices. Thus, we find for the excess free energy due to the interchain attraction a closed expression, again extending the sum over the modes to a series,

$$
\begin{aligned}
\Delta F^{(\mathrm{c})} & =-k_{\mathrm{B}} T \ln \mathcal{Z}_{\mathrm{rel}}^{(\mathrm{c})} \\
& =\frac{k_{\mathrm{B}} T}{2} \sum_{m=1}^{\infty} \ln \left(1+\frac{\tilde{g}}{2} c_{m}^{-1}\right),
\end{aligned}
$$

in agreement with performing the limit $N \rightarrow \infty$ at finite $\tilde{g}$ in Eq. (27).

\section{FORCE-EXTENSION RELATION}

Using Eqs. (27) and (28), straightforward yet tedious differentiation with respect to $f$ yields the force-extension relation

$$
\frac{\langle\Delta x\rangle}{L}=\frac{k_{\mathrm{B}} T g}{8 N f^{2}} \sum_{l=1}^{N-1} \frac{n_{l}\left(\delta_{f}\right)}{d_{l}\left(\delta_{f}, \epsilon_{g}\right)},
$$

with numerator

$$
\begin{aligned}
n_{l}\left(\delta_{f}\right)= & 2\left(\cosh \delta_{f}-\cos \phi_{l}\right)-\left(1-\cos \phi_{l}\right) \\
& \times\left[3 \frac{\sinh \delta_{f}}{\delta_{f}}-\frac{1-\cos \phi_{l} \cosh \delta_{f}}{\cosh \delta_{f}-\cos \phi_{l}}\right]
\end{aligned}
$$

and denominator

$$
\begin{aligned}
d_{l}\left(\delta_{f}, \epsilon_{g}\right)= & \left(1-\cos \phi_{l}\right)\left(\cosh \delta_{f}-\cos \phi_{l}\right) \\
& +\epsilon_{g}\left[\cosh \delta_{f}-\cos \phi_{l}-\left(1-\cos \phi_{l}\right) \frac{\sinh \delta_{f}}{\delta_{f}}\right]
\end{aligned}
$$

in terms of the length ratio $\delta_{f}$ from Eq. (29), the ratio $\epsilon_{g}$ from Eq. (32), and the phases $\phi_{l}$ from Eq. (30) [25].

Since a direct interpretation of the expressions in Eqs. (35) and (36) is difficult, in Fig. 2 we show the calculated force-extension relation for several numbers and strengths of cross-links, as a function of the dimensionless force variable $f_{r}$, Eq. (11). The dimensionless parameter for the cross-link strength is

$$
g_{a}:=\frac{g L^{2}}{k_{\mathrm{B}} T}=2 \frac{L^{2}}{a_{c}^{2}},
$$


FIG. 2. (Color online) Force-extension relation at a ratio of persistence length to contour length $L_{p} / L=10$ : For different numbers of cross-links of strength $g_{a}=50$ (top) and for 10 cross-links of varying strength (bottom).

which, by virtue of our entropic-spring model for the crosslinks, can be expressed as the squared ratio of the WLC contour length and one cross-link's length at rest.

First, in the upper part of Fig. 2, the force-extension curve is plotted for different numbers of cross-links, at constant strength of a single cross-link. The overall form of the saturation curve is reminiscent of an unconstrained weakly bending WLC [12]. Evidently, the general effect of crosslinking is to increase the extension in force direction relative to an uncross-linked weakly bending chain because cross-links effectively suppress thermal fluctuations perpendicular to the aligning force. The growth of the extra alignment with the number of cross-links is nonlinear, the increase relative to a chain pair with less cross-links being largest for a few cross-links, and for weak stretching. The limit of continuous cross-linking, cf. Sec. (III B), is discussed in Sec. IV B.

Enforcing a smaller and smaller cross-link length (increasing the cross-link strength) also enhances the alignment or effective stiffness, as visible in the lower part of Fig. 2, in which the cross-link strength is varied at a fixed number of cross-links. The limit of strong topological constraints at the cross-link sites (hard or inextensible cross-links) is presented in Sec. IV A.

Cross-links are most effective in suppressing transverse fluctuations and aligning the chain pair at small reduced stretching forces, at which the directional memory length $\sqrt{\kappa / f}$ is still large compared to the cross-link spacing $L / N$. For these relatively weak pulling forces, there is a regime of linear elasticity for all numbers and strengths of crosslinks, taken a closer look upon in Sec. IV C. For increasing force, the incremental extension due to cross-links decreases 


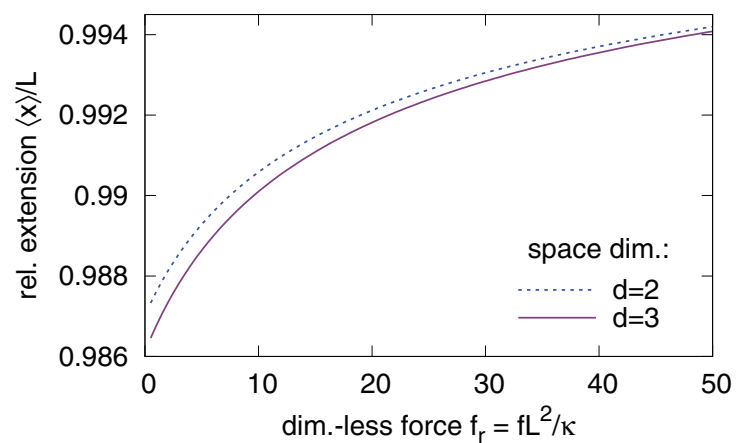

FIG. 3. (Color online) Force-extension relation for 10 cross-links of strength $g_{a}=50$ at $L_{p} / L=10$, in $d=2$ and $d=3$.

since, at strong stretching, the dominant contribution to the saturating extension arises from "pulling out" the remaining length reserves stored in thermal undulations. The asymptotic decay of the cross-link contribution with force is computed in Sec. IV D.

In Fig. 2, we have chosen a ratio of persistence to contour length $L_{p} / L=10$ sufficiently large as to give for all $f_{r}$ relative extensions close to 1 , to explore the entire range of stretching forces and yet keep the weakly bending approximation. A ratio $L_{p} / L$ of this order would apply to long microtubules [26]. The persistence length of actin is about $15 \mu \mathrm{m}$ [26,27], thus for typical lengths of actin filaments in solution, the ratio $L_{p} / L$ is of order 1 . For smaller ratios, e.g., $L_{p} / L \sim 0.1$ for type I collagen fibrils [28,29], or $L_{p} / L \sim 0.01$ for $10 \mu \mathrm{m}$ of double-stranded DNA [11], our predictions are reasonable at strong stretching only.

As mentioned above, the embedding space dimension $d$ can be easily generalized to $d=3$ within the weakly bending approximation. Without accounting for twist, the two coordinates transverse to the stretching force decouple both in the bending or stretching as in the cross-link part of the Hamiltonian, so that for $d=3$ we obtain the square of the partition function from Sec. III and a factor of 2 in the incremental free energy. Note, however, that the factor in the relation between bending rigidity $\kappa$, featuring in the dimensionless force $f_{r}$, and persistence length $L_{p}$, cf. Eq. (2), changes also. In Fig. 3, one set of parameters is evaluated for $d=2$ and $d=3$, respectively. The extension in force direction is slightly decreased in $d=3$ since, independently for each transverse direction, the deviation from maximal extension due to thermal fluctuations is larger in modulus than the aligning cross-link contribution. In the following results, we return to the two-dimensional case.

\section{A. Limit of hard cross-links}

In the limit of infinite cross-link strength or vanishing ratio of cross-link to contour length, $a_{c} / L \rightarrow 0$, we have

$$
\frac{\left\langle\Delta x^{(h)}\right\rangle}{L}=\frac{k_{\mathrm{B}} T}{8 f L} \sum_{l=1}^{N-1} \frac{n_{l}\left(\delta_{f}\right)}{d_{l}^{(h)}\left(\delta_{f}\right)},
$$

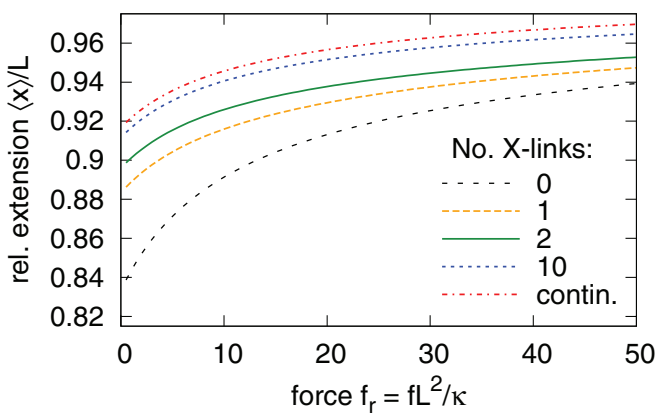

FIG. 4. (Color online) Force-extension relation for different numbers of hard cross-links, at persistence length $L_{p}=L$.

with $n_{l}\left(\delta_{f}\right)$ from Eq. (36a), and

$$
d_{l}^{(h)}\left(\delta_{f}\right)=\cosh \delta_{f}-\cos \phi_{l}-\left(1-\cos \phi_{l}\right) \frac{\sinh \delta_{f}}{\delta_{f}} .
$$

The corresponding force-extension curves are shown in Fig. 4, for more flexible weakly bending chains (with $L_{p}=L$ ) than the rather rod-like chains in Figs. 2 and 5. Since hard crosslinks completely eliminate relative motion of the filaments transverse to stretching at the cross-linking sites, the relative alignment effect due to cross-links is seen to be stronger.

\section{B. Continuous cross-linking}

In this section, we discuss the limit of continuous crosslinking from Sec. III B, $N \rightarrow \infty$ at total strength $\tilde{g}:=N g$. The incremental extension of one chain due to continuous crosslinking is computed from $\Delta F^{(\mathrm{c})}$, Eq. (34), by differentiation, cf. the general expression for all values of $\tilde{g}$ and further remarks in Appendix C. In the case of continuous and rigid crosslinking $\left(a_{c} \rightarrow 0\right)$, the force-extension relation is

$$
\frac{\left\langle x^{(\mathrm{c}, h)}\right\rangle}{L}=1-\frac{L}{4 L_{p}}\left\{\frac{\operatorname{coth} \sqrt{f_{r}}}{\sqrt{f_{r}}}-\frac{1}{f_{r}}\right\} .
$$

Comparing this result to Eq. (10), we observe that the squared thermal $y$ fluctuations, cf. Eq. (9), are reduced by $1 / 2$ relative to the uncross-linked case. This does, however, not imply that the two chains attached to each other rigidly can be treated as unconstrained weakly bending WLCs with just one effective persistence length or bending stiffness $\kappa_{\text {eff }}$ since $f_{r}$ itself depends on the bending stiffness $\kappa$. The different apparent persistence lengths in the force regimes of linear elasticity and

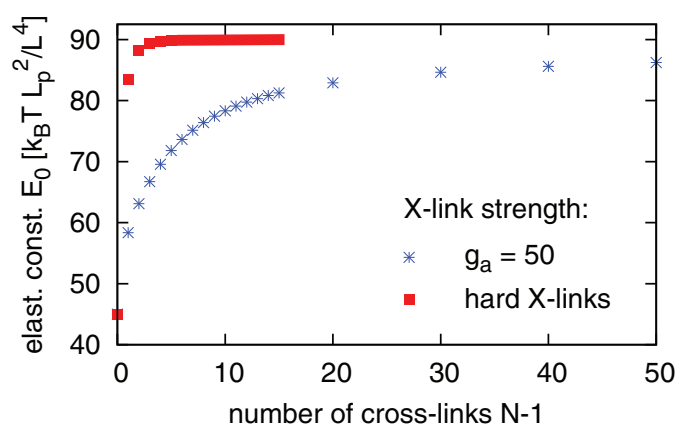

FIG. 5. (Color online) Linear elastic constant as a function of the number of cross-links, $N-1$, at $L_{p} / L=10$. 
of strong stretching are discussed in the next two sections. The extension for rigid, continuous cross-linking for arbitrary force, Eq. (40), is shown as the topmost curve in Fig. 4, corresponding in Fig. 2 to the asymptotic case of both infinite number and strength of cross-links.

For continuous cross-linking of any strength $\tilde{g}$, the modediagonal structure of the total Hamiltonian cf. Eq. (33), allows us to extract a length-scale-dependent apparent stiffness as discussed by the authors of $[17,30]$. The extensions of the uncross-linked and the continuously cross-linked, weakly bending filament, cf. Eqs. (31) and (34), are, respectively,

$$
\begin{gathered}
\langle x\rangle_{\mathcal{H}_{0}}=L-\frac{k_{\mathrm{B}} T}{2} \sum_{m=1}^{\infty} \frac{\partial_{f} c_{m}}{c_{m}}, \\
\left\langle x^{(\mathrm{c})}\right\rangle=L-\frac{k_{\mathrm{B}} T}{2} \sum_{m=1}^{\infty}\left(\frac{\partial_{f} c_{m}}{c_{m}}\right) \frac{\tilde{g}+4 c_{m}}{2\left(\tilde{g}+2 c_{m}\right)}, \\
\text { with } c_{m}=\frac{L q_{m}^{2}\left(f+\kappa q_{m}^{2}\right)}{4}
\end{gathered}
$$

and wave numbers $q_{m}=m \pi / L$. For large $q_{m}$ (small length scales) and finite $\tilde{g}$, the apparent stiffness is the bare local bending stiffness. For small $q_{m}$ (or $\tilde{g} \rightarrow \infty$ ), the apparent stiffness is modified by cross-linking and indeed becomes independent of $\tilde{g}$, so that we recover the force-extension relation of Eq. (40).

\section{Force-free extension and linear response regime}

Knowing the exact extension curve for all values of the force $f$, we can address the equilibrium extension in the limit $f \rightarrow 0$ and the linear elasticity for small $f$ (assuming a large persistence length $L_{p}$, so that weakly bending holds). This linear response or weak-perturbation regime may be the best accessible for stretching experiments on sensitive biopolymers. Moreover, the force-extension curves computed within our model suggest that the chain pair's extension at moderate or zero force, cf. Fig. 2, is most indicative of the degree of cross-linking. Without cross-links, the equilibrium extension of a weakly bending WLC parallel to alignment for $f \rightarrow 0$ is, following Eq. (10),

$$
\frac{\left\langle x_{0}\right\rangle_{\mathcal{H}_{0}}}{L}:=\lim _{f \rightarrow 0} \frac{\langle x\rangle_{\mathcal{H}_{0}}}{L}=1-\frac{L}{6 L_{p}},
$$

cf. [23]. The deviation from the maximal extension is inversely proportional to the persistence length. Cross-links increase the equilibrium extension according to

$$
\frac{\left\langle\Delta x_{0}\right\rangle}{L}=\frac{g_{a} L^{2}}{20 N^{5} L_{p}^{2}} \sum_{l=1}^{N-1} \frac{\left[x_{l}^{2}+13 x_{l}+16\right] /\left(1-x_{l}\right)}{6\left(1-x_{l}\right)^{2}+\frac{2 g_{a} L}{N^{3} L_{p}}\left(2+x_{l}\right)},
$$

with $x_{l}:=\cos \phi_{l}$. For large $L_{p}$ and finite $g_{a}$, this expression is $\mathcal{O}\left(L / L_{p}\right)^{2}$ and hence small. For hard cross-links, Eq. (44) is linear in $L / L_{p}$, so that an effective persistence length $L_{p \text {,eff }}>$ $L_{p}$ of the cross-linked chains can be defined, viz.,

$$
\frac{L_{p}}{L_{p, \mathrm{eff}}}=1-\frac{3}{20 N^{2}} \sum_{l=1}^{N-1} \frac{x_{l}^{2}+13 x_{l}+16}{\left(1-x_{l}\right)\left(2+x_{l}\right)} .
$$

In the limit of continuous, rigid cross-linking discussed in Sec. IV B, the increase in equilibrium extension is

$$
\frac{\left\langle\Delta x_{0}^{(\mathrm{c}, h)}\right\rangle}{L}=\frac{L}{12 L_{p}} .
$$

Upon comparison with Eq. (43), we thus find the zero-force extension of one weakly bending WLC with twice the original persistence length or $\kappa_{\text {eff }}=2 \kappa$.

In Fig. 5, we show the dependence of the linear elastic constant $E_{0}$,

$$
E_{0}^{-1}:=\left.\partial_{f}\langle x\rangle\right|_{f=0},
$$

on the number of cross-links. For extensible cross-links, a large increase with cross-link number up to $N \approx 10$ is followed by a saturation to twice the elastic constant of an uncross-linked weakly bending WLC. For hard cross-links, the increase in the elastic constant caused by introducing only a few crosslinks is even more drastic, and the curve approaches a step function. The elastic constant of an uncross-linked weakly bending WLC, $E_{0}=45 k_{\mathrm{B}} T L_{p}^{2} / L^{4}$ in $d=2$, scales with the contour length $L$ as $L^{-4}$. Asymptotically for large $L$, this remains the dominant contribution also with a finite number of extensible cross-links since the lowest order of $\partial_{f}\langle\Delta x\rangle$ in $L$ is $\propto g L^{7} / L_{p}^{3}$, cf. Eq. (35). For hard cross-links, in contrast, the derivatives of the cross-link terms are $\propto L^{4} / L_{p}^{2}$, so that their contribution to the elastic constant is no longer subdominant in $1 / L$. As mentioned earlier, the limit of continuous, rigid cross-linking yields twice the elastic constant of an uncrosslinked weakly bending WLC and thus shows the same scaling with $L^{-4}$.

\section{Strong stretching limit}

Here, we consider the limit of strong stretching, i.e., $f_{r} \gg 1$, or for finite $N, \sqrt{f_{r}} / N \gg 1$, which means that the directional memory length introduced after Eq. (29) is much smaller than the cross-link spacing,

$$
\sqrt{\frac{\kappa}{f}} \ll \frac{L}{N}
$$

At finite cross-link strength and for a finite number of crosslinks, the dependence on the individual eigenvector phases remains in the limit of strong stretching, yet the asymptotic scaling with $f_{r}$ is the same for all summands,

$$
\frac{\langle\Delta x\rangle}{L}=\frac{g_{a} L^{2}}{N L_{p}^{2}} f_{r}^{-2} \sum_{l=1}^{N-1} \frac{1}{1-\cos \phi_{l}}+\mathcal{O}\left(f_{r}^{-5 / 2}\right) .
$$

The same asymptotic decay ensues for continuous crosslinking at finite total strength $\tilde{g}_{a}:=2 N L^{2} / a_{c}^{2}$, cf. Eq. (C1), but with a simpler coefficient,

$$
\frac{\left\langle\Delta x^{(\mathrm{c})}\right\rangle}{L}=\frac{\tilde{g}_{a} L^{2}}{3 L_{p}^{2}} f_{r}^{-2}+\mathcal{O}\left(f_{r}^{-5 / 2}\right) .
$$

In the presence of a finite number of hard cross-links, strong stretching asymptotically results in an extension increment, which is independent of the individual eigenvector phases and 
decays proportional to $f_{r}^{-1}$,

$$
\frac{\left\langle\Delta x^{(h)}\right\rangle}{L}=\frac{(N-1) L}{2 L_{p}} f_{r}^{-1}+\mathcal{O}\left(f_{r}^{-3 / 2}\right) .
$$

Hence the asymptotic decay of the extra alignment with force is markedly slower than for extensible cross-links. In all cases mentioned so far, the impact of cross-linking diminishes fast for strong stretching, and the extension curve displays the saturation $\propto f^{-1 / 2}$ of the uncross-linked chain's extension to the contour length.

Obviously, the asymptotic behavior computed for hard cross-links in Eq. (51) cannot apply in the limit $N \rightarrow \infty$ of continuous, rigid cross-linking, due to the diverging prefactor $\propto(N-1)$. Indeed, in this case, the asymptotic decay of the inter-chain contribution to the extension is even slower, viz.

$$
\frac{\left\langle\Delta x^{(\mathrm{c}, h)}\right\rangle}{L}=\frac{L}{4 L_{p}} f_{r}^{-1 / 2}+\mathcal{O}\left(f_{r}^{-1}\right) .
$$

This is the same scaling as the saturation of the uncross-linked chain's extension, hence the stabilizing effect of continuous, rigid cross-linking is manifest even for large stretching forces. Moreover, in the strong stretching limit, a continuously and rigidly linked chain behaves effectively like a weakly bending WLC with fourfold original persistence length or $\kappa_{\text {eff }}=4 \kappa$.

Of course, the WLC picture is oversimplified at very strong stretching, at which inextensibility is clearly violated for many semiflexible biopolymers [2,31].

\section{E. Differential stiffness}

A quantity of interest related to the $x$ extension is the differential stiffness: In the corresponding experiment for our system, both fibers are prestressed by longitudinal stretching, subsequently, the strain response to a small change in the applied stress is measured. From the force-extension relation, we can readily compute the differential stiffness as the quotient of force and extension increment at a given prestretching force

$$
E^{-1}(f):=\partial_{f}\langle x\rangle,
$$

generalizing the elastic constant discussed in Sec. IV C.

In an effort to highlight the effective stiffening due to cross-links, we show in Fig. 6 the increase in differential



FIG. 6. (Color online) Increase in differential stiffness due to hard cross-links relative to a weakly bending WLC without cross-links, at $L_{p} / L=10$. stiffness relative to uncross-linked weakly bending WLCs for the case of hard cross-links. Again, for a few cross-links, the differential stiffness is particularly enhanced at weak stretching. Already for a single, hard cross-link, the linear elastic constant is increased by more than $80 \%$ compared to the uncross-linked case. Upon approaching the limit of rigid, continuous cross-linking, cf. Eq. (40), the differential stiffness is increased by a factor of 2 for all values of $f_{r}$.

\section{DISCUSSION AND OUTLOOK}

Within a transparent mesoscopic model of cross-linked polymers, the elasticity of two irreversibly cross-linked WLCs subjected to a tensile force has been studied in the weakly bending approximation. The validity of the later is granted by assuming either a large tensile force or a large bending rigidity. For an arbitrary number of cross-links with given strength, we have calculated the free energy and, derived thereof, the force-extension relation exactly. Both with increasing number $N$ and with increasing strength $g$ of the crosslinks, the effective stiffness of the chain pair increases since cross-links stabilize the chains against thermal undulations. Particularly for weak stretching, the enhancement in alignment is considerable, such that in corresponding weak-perturbation experiments on biopolymers, the increase in the linear elastic constant may be a useful indicator of (partial) crosslinking. As expected, the effect is most pronounced for hard cross-links.

In the limit of strong pulling forces, the additional extension $\langle\Delta x\rangle$ due to cross-linking decreases, and the elasticity of an uncross-linked WLC [12] dominates. However, the asymptotic behavior for large stretching forces is different for hard and extensible cross-links, as well as for discrete and continuous cross-linking, and is summarized in Table I. For extensible cross-links, the cross-link contribution decays as $f^{-2}$, for a finite number of hard cross-links, as $f^{-1}$. A slower decay is found in the limit of both cross-link number $N \rightarrow \infty$ and cross-link strength $g \rightarrow \infty$, in which the two chains are linked continuously and rigidly along their contour: For strong stretching, the asymptotic form of the force-extension relation reflects the behavior of one uncross-linked weakly bending chain with effective persistence length $4 L_{p}$ and with the known $f^{-1 / 2}$ scaling.

From the exact extension for all stretching forces, we have computed another experimentally relevant observable, viz., the differential stiffness of the (prestretched) cross-linked chains. Even a small number of cross-links enhances the differential stiffness dramatically. Again, the impact is largest for small stretching forces, which can be considered within the weakly

TABLE I. Exponents of the asymptotic scaling of $\langle\Delta x\rangle$ with force $f_{r}=f L^{2} / \kappa$ in the strong stretching limit $f_{r} \gg 1$.

\begin{tabular}{lcc}
\hline \hline & \multicolumn{2}{c}{ Number of cross-links } \\
\cline { 2 - 3 } & Finite & Infinite \\
\hline Extensible & -2 & -2 \\
Hard & -1 & $-1 / 2$ \\
\hline \hline
\end{tabular}


bending approximation for WLCs with a large persistence length $L_{p} / L$.

Several generalizations of our approach are possible: As alluded to in [16], our model is not in principle restricted to a pair of cross-linked filaments, but should be generalizable to describe the tensile elasticity of a stretched weakly bending WLC bundle, possibly with random and/or reversible cross-links. To take into account the nonaffine deformation of cross-linked bundles, we may have to consider also the shearing of cross-links. Detailed analysis of bundles exists due to the relevance for actin networks, mostly for reversibly cross-linked and extensible, semiflexible polymers [30,32]. Particularly for bundles, the effect of excluded volume interaction, neglected in this work, remains to be explored.

Apart from the activities in this realm, major recent research efforts are devoted to the impact of structural inhomogeneities caused by the (local) breaking of complimentary base-pair bonds ("unzipping" or denatured "bubbles") on the elasticity of double-stranded DNA $[33,34]$. A class of semimicroscopic models convenient for analyzing the thermal denaturation transition, as well as the "bubble" statistics and dynamics [35], focuses on the form of the base-pairing interaction, but does not account for the polymers' conformational degrees of freedom, which determine certain DNA properties [36]. In the breathing DNA model [37], two discrete chains (consisting of interacting "beads") with bending and stretching rigidity interact via the pairing energy of complimentary bases, represented by a Morse potential. Another semimicroscopic model, amenable to a transfer matrix method, considers a discrete WLC model for the chain conformations, coupled to an one-dimensional Ising model describing the internal base-pair states [38]. In the context of denaturation of DNA, it would be interesting to extend our model to reversible cross-linking to study the coexistence of "ladders" (cross-linked strand sections) and "bubbles" (open sections). Since the persistence length of single-stranded DNA is comparatively small, the model of weakly bending chains can be employed in the case of large longitudinal pulling forces only.

A first, obvious step towards this direction will be to address with our method two parallel aligned WLCs whose arc length is sectioned into cross-linked and disconnected parts. In the setup we have considered here, all ingredients, viz. cross-linking, bending stiffness, and longitudinal forcing, act to decrease transverse fluctuations of the chains. An unzipping transition could presumably be studied in an altered situation, e.g., one, in which the cross-linked filaments are teared apart at one end. More complicated refinement of our model might account for twist and overstretching, effects shown to be essential for the elasticity of DNA [39].

\section{ACKNOWLEDGMENTS}

We thank W. T. Kranz for valuable discussions and a careful reading of the manuscript. Financial support by the Deutsche Forschungsgemeinschaft through Grants No. SFB937/A1 and No. SFB-937/A4 is gratefully acknowledged. P. B. acknowledges support by Kyungpook National University Research Fund, 2012.

\section{APPENDIX A: STRUCTURE OF THE PROJECTOR SUM $P$}

The matrix representation of the cross-link projector sum $P$, cf. Eqs. (25), has the following structure:

$$
\begin{aligned}
P & =\left(\begin{array}{ccc}
1 & 1 & \ldots \\
1 & 1 & \\
\vdots & & \ddots
\end{array}\right) \\
& \left(\begin{array}{ccccccccc}
1 & 0 & \ldots & 0 & \ldots & 0 & -1 & 0 \\
0 & \ddots & & \vdots & & . \cdot & 0 & \vdots \\
\vdots & 0 & 1 & & -1 & 0 & \vdots & \\
0 & \ldots & & 0 & & \ldots & 0 & \\
\vdots & 0 & -1 & & 1 & 0 & \vdots & \\
0 & . \cdot & & \vdots & & \ddots & 0 & \\
-1 & 0 & \ldots & 0 & \ldots & 0 & 1 & \\
0 & \ldots & & & & & & 0
\end{array}\right) .
\end{aligned}
$$

\section{APPENDIX B: TRACE OF POWERS OF THE PROJECTOR SUM}

By decomposing mode indices $m \in\{1,2, \ldots\}$ according to the block structure into

$$
\begin{aligned}
m & =2 \mu N-r, \text { with the definitions } \\
\mu & :=\left\lceil\frac{m}{2 N}\right\rceil \in\{1,2, \ldots\} \text { (block index), } \\
r & \in\{0, \ldots, 2 N-1\} \text { (index within a block), } \\
\rho(r) & :=\left\lfloor\frac{r}{N}\right\rfloor \in\{0,1\} \text { (quadrant within a block), }
\end{aligned}
$$

the entries of $P$, cf. Eqs. (25) and (A1), can be encoded in product form, the first two factors indicating the location of nonzero entries, the last factor the sign

$$
\begin{aligned}
P_{m_{1} m_{2}} & =\delta_{m_{1}-m_{2}, 2 \mathbb{Z} N}-\delta_{m_{1}+m_{2}, 2 \mathbb{Z} N} \\
& =\left(\delta_{r_{1}, r_{2}}+\delta_{r_{1}, 2 N-r_{2}}\right)\left(1-\delta_{r_{1}, \mathbb{Z} N}\right)(-1)^{\rho_{1}+\rho_{2}} .
\end{aligned}
$$

Then, with the diagonal matrix $C$, cf. Eq. (18), we write the trace of a power $k$ of $C^{-1} U U^{T}$, cf. Eq. (23), as

$$
\begin{aligned}
\left(\frac{g N}{2}\right)^{-k} \operatorname{tr}\left(C^{-1} U U^{T}\right)^{k} \\
=\sum_{m_{1}, \ldots, m_{k}=1}^{\infty} c_{m_{1}}^{-1}\left(\delta_{m_{1}-m_{2}, 2 \mathbb{Z} N}-\delta_{m_{1}+m_{2}, 2 \mathbb{Z} N}\right) \cdot \ldots \\
\quad \times c_{m_{k}}^{-1}\left(\delta_{m_{k}-m_{1}, 2 \mathbb{Z} N}-\delta_{m_{k}+m_{1}, 2 \mathbb{Z} N}\right) \\
=\sum_{\mu_{1}, \ldots, \mu_{k}=1}^{\infty} \sum_{r_{1}, \ldots, r_{k}=0}^{2 N-1} \\
\quad \times c_{2 \mu_{1} N-r_{1}}^{-1}\left(\delta_{r_{1}, r_{2}}+\delta_{r_{1}, 2 N-r_{2}}\right)\left(1-\delta_{r_{1}, \mathbb{Z} N}\right)(-1)^{\rho_{1}+\rho_{2}} \\
\quad \times c_{2 \mu_{2} N-r_{2}}^{-1}\left(\delta_{r_{2}, r_{3}}+\delta_{r_{2}, 2 N-r_{3}}\right)\left(1-\delta_{r_{2}, \mathbb{Z} N}\right)(-1)^{\rho_{2}+\rho_{3}} \\
\quad \times \ldots \\
\quad \times c_{2 \mu_{k} N-r_{k}}^{-1}\left(\delta_{r_{k}, r_{1}}+\delta_{r_{k}, 2 N-r_{1}}\right)\left(1-\delta_{r_{k}, \mathbb{Z} N}\right)(-1)^{\rho_{k}+\rho_{1}} .
\end{aligned}
$$

Due to the symmetry of the constraints, we are left with the summation over one of the $r_{j}$ without 0 and $N$. If we split 
this sum according to the constraints and to the eigenvector structure mentioned above as

$$
\sum_{r=0}^{2 N-1} f_{r}\left(1-\delta_{r, \mathbb{Z} N}\right)=\sum_{l=1}^{N-1}\left(f_{l}+f_{2 N-l}\right),
$$

we finally arrive at

$$
\begin{aligned}
\left(\frac{g N}{2}\right)^{-k} \operatorname{tr}\left(C^{-1} U U^{T}\right)^{k} \\
=\sum_{\mu_{1}, \ldots, \mu_{k}=1}^{\infty} \sum_{l=1}^{N-1}\left(c_{2\left(\mu_{1}-1\right) N+l}^{-1}+c_{2 \mu_{1} N-l}^{-1}\right) \cdot \ldots \\
\quad \times\left(c_{2\left(\mu_{k}-1\right) N+l}^{-1}+c_{2 \mu_{k} N-l}^{-1}\right) \\
=\sum_{l=1}^{N-1}\left[\sum_{\mu=1}^{\infty}\left(c_{2(\mu-1) N+l}^{-1}+c_{2 \mu N-l}^{-1}\right)\right]^{k} .
\end{aligned}
$$

\section{APPENDIX C: CONTINUOUS CROSS-LINKING}

Here, we present the general result for the limit of continuous cross-linking dealt with in Secs. III B and IV B. The incremental extension due to this kind of cross-linking computed from Eq. (34) is

$$
\frac{\left\langle\Delta x^{(\mathrm{c})}\right\rangle}{L}=\frac{L}{4 L_{p}}\left\{\frac{\frac{\sin g^{-}}{g^{-}}-\frac{\sinh g^{+}}{g^{+}}}{\cosh g^{+}-\cos g^{-}}+\frac{\operatorname{coth} \sqrt{f_{r}}}{\sqrt{f_{r}}}-\frac{1}{f_{r}}\right\},
$$

as a function of the dimensionless force $f_{r}$, Eq. (11), and the dimensionless parameters

$$
\begin{aligned}
& g^{-}:=\sqrt{2 \sqrt{2 \tilde{g} L^{3} / \kappa}-f_{r}}, \\
& g^{+}:=\sqrt{2 \sqrt{2 \tilde{g} L^{3} / \kappa}+f_{r}},
\end{aligned}
$$

which apart from $f_{r}$ contain the ratio of total interchain attraction $\tilde{g} L^{2}=2 N k_{\mathrm{B}} T L^{2} / a_{c}^{2}$ to bending energy $\kappa / L$.

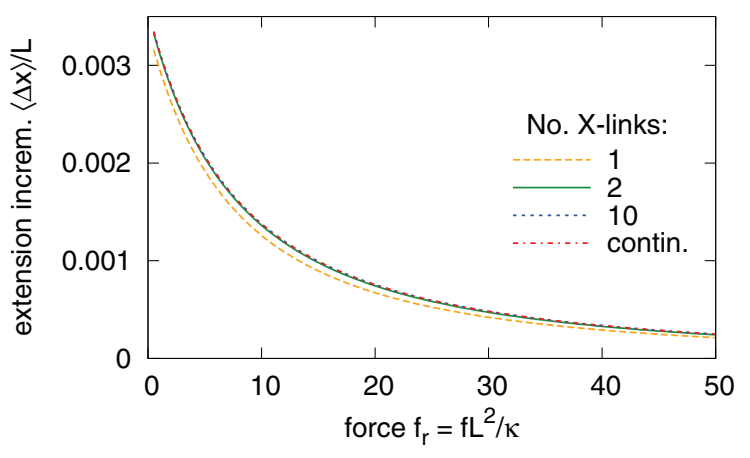

FIG. 7. (Color online) Cross-link-induced extension increment at total cross-link strength $\tilde{g}_{a}=500$ and $L_{p} / L=10$.

[The function in Eq. (C1) is a continuous, real-valued function of $f_{r}$ independently of the sign of the outer square root's argument.]

Comparing the force-extension curves for finite numbers of cross-links to each other and to those for continuous cross-linking, we find an approximate collapse of all curves with the same total inter-chain attraction $\tilde{g}_{a}=2 N L^{2} / a_{c}^{2}$, as shown in Fig. 7. Except for the case of a single cross-link, the force-extension relation is over a large range of forces to high numerical precision determined by the product of cross-link number +1 and strength of a single cross-link only. On the basis of Eqs. (34) and (27), this apparent scaling can be traced back to the rapid decay of the coefficients $c_{m}^{-1}$ with mode index $m$. The sum over eigenvectors $l$ in Eq. (27) for finite $N$ is dominated by those with entries at the lowest modes, and of the set of modes represented by one eigenvector, only the lowest mode gives an appreciable contribution. Thereby, the "missing" modes $m=\mathbb{Z} N$ and, finally, the deviation from the series in Eq. (34) are negligible but for very small $N$. According to the slower decay $\propto m^{-2}$ of the stretching contribution to the coefficients $c_{m}^{-1}$, the approximate scaling must break down for strong stretching (and small persistence lengths). In this regime, however, the additional extension due to cross-links is a small quantity anyway.
[1] C. Bustamante, J. F. Marko, E. D. Siggia, and S. Smith, Science 265, 1599 (1994).

[2] X. Liu and G. H. Pollack, Biophys. J. 83, 2705 (2002).

[3] M. L. Tanzer, Science 11, 561 (1973).

[4] D. R. Eyre and J.-J. Wu, in Collagen, Top. Curr. Chem., edited by J. Brinckmannn, H. Notbohm, and P. K. Müller, Springer, Berlin, (2005), Vol. 247, pp. 207-229.

[5] J. S. Graham, A. N. Vomund, C. L. Phillips, and M. Grandbois, Exp. Cell Res. 299, 335 (2004).

[6] J. A. J. van der Rijt, K. O. van der Werf, M. L. Bennink, P. J. Dijkstra, and J. Feijen, Macromol. Biosci. 6, 697 (2006).

[7] L. Yang, K. O. van der Werf, B. F. Koopman, V. Subramaniam, M. L. Bennink, P. J. Dijkstra, and J. Feijen, J. Biomed. Mater. Res. 82A, 160 (2007).

[8] P. Benetatos and A. Zippelius, Phys. Rev. Lett. 99, 198301 (2007).
[9] O. Lieleg, M. M. A. E. Claessens, and A. R. Bausch, Soft Matter 6, 218 (2010).

[10] M. L. Gardel, J. H. Shin, F. C. MacKintosh, L. Mahadevan, P. Matsudaira, and D. A. Weitz, Science 304, 1301 (2004).

[11] C. Bustamante, S. B. Smith, J. Liphardt, and D. Smith, Curr. Opin. Struct. Biol. 10, 279 (2000).

[12] J. F. Marko and E. D. Siggia, Macromolecules 28, 8759 (1995).

[13] O. Kratky and G. Porod, Recl. Trav. Chim. Pays-Bas 68, 1106 (1949).

[14] N. Saitô, K. Takahashi, and Y. Yunoki, J. Phys. Soc. Jpn. 22, 219 (1967).

[15] A. Y. Grosberg and A. R. Khokhlov, Statistical Physics of Macromolecules, AIP Series in Polymers and Complex Materials (New York, 1994).

[16] P. Benetatos, S. Ulrich, and A. Zippelius, New J. Phys. 14, 115011 (2012). 
[17] R. Everaers, R. Bundschuh, and K. Kremer, Europhys. Lett. 29 , 263 (1995).

[18] T. B. Liverpool, R. Golestanian, and K. Kremer, Phys. Rev. Lett. 80, 405 (1998).

[19] C. Heussinger, F. Schüller, and E. Frey, Phys. Rev. E 81, 021904 (2010).

[20] P. Benetatos and E. M. Terentjev, Phys. Rev. E 81, 031802 (2010).

[21] P. Benetatos and E. M. Terentjev, Phys. Rev. E 84, 022801 (2011).

[22] J. Kierfeld, O. Niamploy, V. Sa-yakanit, and R. Lipowsky, Eur. Phys. J. E 14, 17 (2004).

[23] P. K. Purohit, M. E. Arsenault, Y. Goldman, and H. H. Bau, Int Journal Non-Linear Mech. 43, 1056 (2008).

[24] There are several possibilities to compute the force-extension relation of this model with a quadratic-form Hamiltonian. In [16], for the case of a single cross-link, the matrix was inverted using the Sherman-Morrison formula, an approach which becomes increasingly involved for larger numbers of cross-links.

[25] For a single cross-link, $\cos \phi_{1}=0$, and the expressions in Eqs. (36) simplify considerably, cf. Eq. (9) in [16].

[26] F. Gittes, B. Mickey, J. Nettleton, and J. Howard, J. Cell Biol. 120, 923 (1993).
[27] A. Ott, M. Magnasco, A. Simon, and A. Libchaber, Phys. Rev. E 48, R1642 (1993).

[28] Y.-L. Sun, Z.-P. Luo, A. Fertala, and K.-N. An, Biochem. Biophys. Res. Commun. 295, 382 (2002).

[29] L. Bozec and M. Horton, Biophys. J. 88, 4223 (2005).

[30] C. Heussinger, M. Bathe, and E. Frey, Phys. Rev. Lett. 99, 048101 (2007).

[31] S. B. Smith, Y. Cui, and C. Bustamante, Science 271, 795 (1996).

[32] O. Lieleg, M. M. A. E. Claessens, C. Heussinger, E. Frey, and A. R. Bausch, Phys. Rev. Lett. 99, 088102 (2007).

[33] A. Hanke, M. G. Ochoa, and R. Metzler, Phys. Rev. Lett. 100, 018106 (2008).

[34] D. Marenduzzo, A. Maritan, E. Orlandini, F. Seno, and A. Trovato, J. Stat. Mech. (2009) L04001.

[35] N. Theodorakopoulos, J. Nonlin. Math. Phys. 18, 419 (2011).

[36] N. Theodorakopoulos and M. Peyrard, Phys. Rev. Lett. 108, 078104 (2012).

[37] O.-chul Lee and W. Sung, Phys. Rev. E 85, 021902 (2012).

[38] J. Palmeri, M. Manghi, and N. Destainville, Phys. Rev. E 77, 011913 (2008).

[39] P. Gross, N. Laurens, L. B. Oddershede, U. Bockelmann, E. J. G. Petermann, and G. J. L. Wuite, Nat. Phys. 7, 731 (2011). 